\title{
RM32
}

\section{Stochastic Seismic Inversion in Reservoir} Modeling

A.M. Francis* (Earthworks Environment \& Resources) 
Stochastic seismic inversion has been available as a technique since the paper published by Haas and Dubrule (1994). Early approaches were restricted by computer runtime performance considerations but algorithmic improvements have largely removed this barrier.

However, despite a number of technical advantages stochastic inversion is not routinely used as a constraint in reservoir modeling even though several algorithms are commercially available in popular reservoir modeling software. This may be due in part to user awareness and training but we should also consider to what extent stochastic seismic inversion is capturing the full geological uncertainty.

Current stochastic seismic inversion algorithms which are capable of being run on a desktop workstation are generally based on 3D spatial constraints imposed using variogram models. The variogram has limitations as an appropriate spatial description for some geological environments but the alternative spatial descriptions are computationally expensive to implement for stochastic inversion. An efficient point-by-point algorithm which incorporates better geological spatial description has been an unsolved requirement for reservoir modeling for the last 15 years. The estimation of horizontal range and anisotropy parameters for the variogram is problematic. The common practice of using horizon slices through coloured inversion results always seems to imply horizontally isotropic variograms with short ranges. The generation of probability cubes classified from stochastic inversion and their use to constrain reservoir models is the currently accepted practice that addresses some of these concerns.

Obtaining prior information on non-stationary trends is also important both for inversion and generally for reservoir modeling. It is common to make the assumption laterally that the model is stationary both for mean and variance. For a complete uncertainty description the low frequency must be simulated as well as the high frequency in a stochastic inversion and an example illustrating the magnitude of the low frequency uncertainty and its implication for subsequent property prediction is shown.

In the context of reservoir modeling both coloured/relative and deterministic inversion suffer from limitations related to scale change and resolution and, in the case of deterministic inversion, insurmountable problems introduced by the low frequency model which preclude deterministic inversion from use in reservoir modeling. In contrast, stochastic seismic inversion has several advantages as a means to condition reservoir models with seismic properties, particularly in addressing scale change, spatial variability and as a workflow for introducing the seismic properties and their associated uncertainty to the reservoir model. Despite its limitations stochastic inversion is still currently the best tool available for introducing seismic property constraints to reservoir modeling. A typical workflow for using stochastic inversion in reservoir modeling and as a standalone uncertainty tool will be used to illustrate some of these applications. 